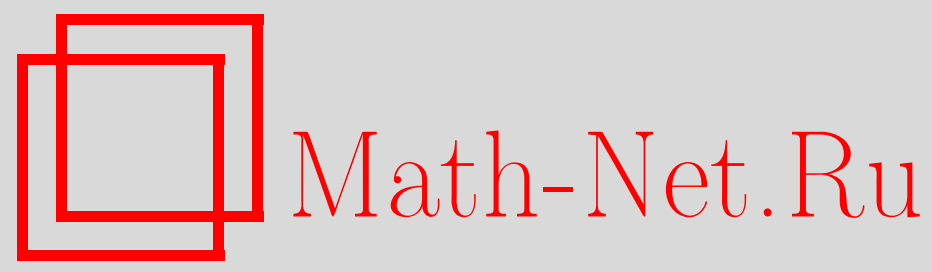

А. Ю. Беляев, Г. А. Чечкин, Усреднение операторов с мелкомасштабной структурой граничных условий, Матем. заметки, 1999, том 65, выпуск 4, 496-510

DOI: https://doi.org/10.4213/mzm1076

Использование Общероссийского математического портала Math-Net.Ru подразумевает, что вы прочитали и согласны с пользовательским соглашением http://www . mathnet.ru/rus/agreement

Параметры загрузки:

IP : 54.237 .59 .107

26 апреля 2023 г., 14:58:08 


\title{
УСРЕДНЕНИЕ ОПЕРАТОРОВ С МЕЛКОМАСШТАБНОЙ СТРУКТУРОЙ ГРАНИЧНЫХ УСЛОВИЙ
}

\author{
А. Ю. Беляев, Г. А. Чечкин
}

\begin{abstract}
В настоящей работе рассматриваются вопросы усреднения краевых задач, содержаших малый параметр в граничных условиях. На основе введенных новых понятий доказывается теорема о компактности семейства решений. На примере задачи с быстро меняющимися граничньми условиями в случае вероятностного описания структуры области проверяется действенность предлагаемого метода исследования.

Библиограффия: 36 названий.
\end{abstract}

Введение. При описании физических процессов уравнениями в частных производных нередко возникают трудности, связанные с выбором граничных условий, адекватных конкретной прикладной задаче. Причины этих трудностей могут происходить из-за геометрической нерегулярности границ области, в которой происходит процесс, или из-за вырождения коэффициентов уравнений вблизи края этой области. Обычно в таких случаях удается описать процесс некоторой регуляризованной задачей с болеепростой структурой граничных условий, в которой учет краевых осцилляций и вырождений производится приближенно с помощью ряда эффективных параметров. Для обоснования такого приближенного подхода требуется асимптотический анализ исходной проблемы, содержащей естественные малые параметры. Возникающий класс асимптотических задач методологически близок к теории усреднения уравнений в перфорированных областях и (или) с быстро осциллирующими коэффициентами (см. [1], [2]).

Проблема усреднения уравнений в областях с быстро осциллирующей границей рассматривалась во многих работах (см. [3]-[16]). В большинстве таких задач главньй член асимптотики решения удовлетворяет исходньгм уравнениям в области со “сглаженной” границей. Граничные условия при этом обычно сохраняют первоначальный вид, но в них появляются усредненные параметры, отражающие геометрию мелкомасштабных осцилляций. Интересный подход к асимптотическому исследованию эллиптических операторов с коэффициентами, вырождающимися вблизи границы области, предложен в [17]. В этом случае тип граничных условий предельной задачи может не совпадать с исходньм. Он зависит от характера вырождения констант эллиптичности.

Работа выполнена при частичной поддержке Российского фонда фундаментальных исследований, грант № 95-01-01232 (первый автор) и грант № 98-01-00062 (второй автор). 
Предлагаемой статье предшествовали работы [18]-[26], где исследовалась проблема усреднения уравнения Пуассона с осциллирующим типом граничных условий. На гранище выделялось подмножество, имеющее в локальных координатах мелкомасштабную периодическую структуру, и на нем ставилось условие Дирихле; на оставшейся части гранищы ставилось условие Неймана. Также были рассмотрены задачи с быстрым непериодическим изменением типа граничных условий [19]-[22], [27], [28] в том числе и для системы теории упругости [22], [27], [28]. В приложениях такие задачи могут возникать при расчетах экранирующих свойств защитных металлических сеток в электромагнитных полях, при исследовании колебания тел, частично закрепленных на гранище. Асимптотика решения в этом случае принципиально зависит от отношения площадей, приходящихся на выделенное подмножество и его дополнение. В частности, если это отношение в периодическом случае не стремится к 0 , то предельная задача для главного члена асимптотики оказьвается задачей Дирихле. Здесь мы исследуем проблему усреднения в случае, когда условие Дирихле для оператора Лапласа выставлено на подмножестве гранищы, необязательно обладающей периодической структрой. Предлагаемый подход включает в себя большой класс задач, в которых для выделенного подмножества может быть разумным образом определен мальй характерный линейный масштаб. В качестве примера мы приводим процедуру построения предельной задачи, ког да геометрия указанного подмножества случайна. При таком обобщении периодической задачи приходится ограничиться исследованием слабых асимптотик решений вблизи границы, в то время как в периодическом случае возможно построение полного асимптотического разложения, включающего в себя корректоры в виде функций, экспоненциально затухающих при удалении от границы (см., например, [23], [25]).

Краевые задачи для Лапласиана сводятся к интегральньп уравнениям на границе области, поэтому рассматриваемую задачу можно трактовать как проблему усреднения некоторого эллиптического псевдодифференциального оператора в перфорированной области. В этом смысле ее исследование является обобщением подхода, развитого в [1], [29], [30] для усреднения уравнений в перфорированных областях случайной структуры.

Кратко результаты о граничном усреднении задач со случайной структурой изложены в [31].

1. Теорема о компактности. В ограниченной области $D \subset \mathbb{R}^{n}, n \geqslant 2$, с достаточно гладкой границей рассматривается семейство краевых задач, зависящих от малого параметра $\varepsilon>0$ :

$$
\begin{gathered}
\Delta u_{\varepsilon}=0 \text { в } D, \\
u_{\varepsilon}=0 \text { на } \Gamma_{\varepsilon} \subset \partial D, \\
\varepsilon \frac{\partial u_{\varepsilon}}{\partial \nu}=g(x) \text { на } \partial D \backslash \Gamma_{\varepsilon} .
\end{gathered}
$$

Здесь $\Gamma_{\varepsilon}-$ часть границы $\partial D$ области $D$, имеющая ячеистую структуру с характерным масштабом $\varepsilon$. На этой части границы задано однородное условие Дирихле. На оставшейся части выставлено условие Неймана, в котором $g(x)$ не зависит от малого параметра, а $\nu$ есть внешняя нормаль к $\partial D$.

Принципиальную роль в асимптотике решения $u_{\varepsilon}$ при $\varepsilon \rightarrow 0$ играет зависимость $\Gamma_{\varepsilon}$ от малого параметра, а сомножитель $\varepsilon$, явно входящий в (1), можно было бы исключить 
из задачи заменой. Он введен для того, чтобы главньй член асимптотики $u_{\varepsilon}$ при $\varepsilon \rightarrow 0$ был порядка 1. Термин характерный масштаб для конкретных примеров ячеистых структур обычно не вызывает затруднений. В частности, если двумерное множество состоит из большого количества одинаковых "пятен", разбросанных равномерно, и доля площади, занятой “пятнами”, не близка к 0 и 1, то в качестве характерного масштаба можно выбрать диаметр “пятна". Однако этот термин мало пригоден для получения результатов достаточной степени общности. Поэтому мы сформулируем основное свойство структур, рассматриваемых в работе, не используя явно геометрических параметров.

ОПРЕДЕЛЕНИЕ 1. Семейство замкнутых множеств $\Gamma_{\varepsilon} \subset \partial D$ назовем самоподобным, если найдутся не зависящие от $\varepsilon$ константы $C_{1}$ и $s, C_{1}>0,1<s \leqslant 2$, такие, что для любого $\varepsilon, 0<\varepsilon \leqslant \varepsilon_{0}$, и любой гладкой функции $\varphi \in C^{\infty}(\bar{D})$ с носителем, не пересекающимся с $\Gamma_{\varepsilon}$, выполнено неравенство

$$
\left(\int_{\partial D}|\varphi|^{s} d x\right)^{1 / s} \leqslant C_{1}\left(\varepsilon \int_{D}(\nabla \varphi)^{2} d x\right)^{1 / 2} .
$$

Ниже будет проиллюстрирована связь свойства самоподобности с эвристическим понятием “характерный масштаб ячеистой структуры”.

Предварительно отметим, что если на многообразии $\partial D$ имеется "пятно" фиксированных размеров, с которым у $\Gamma_{\varepsilon}$ при $\varepsilon \rightarrow 0$ нет общих точек, то (2) не имеет места. Таким образом, самоподобные семейства должны при малых $\varepsilon$ покрьвать $\partial D$ достаточно густо. Неравенство (2) характеризует эту густоту, неявно ограничивая сверху максимальные размеры "пятен" на $\partial D$, оставшихся непокрытыми множествами $\Gamma_{\varepsilon}$.

Наряду со свойством (2) мы будем пользоваться неравенством Пуанкаре в следующей формулировке.

НЕРАВЕНСТВо ПуАНКАРЕ. Для всех функиий, заданных в области D, справедливо неравенство

$$
\int_{D} \varphi^{2} d x \leqslant C_{2}\left(\left(\int_{\partial D}|\varphi| d x\right)^{2}+\int_{D}(\nabla \varphi)^{2} d x\right),
$$

әде константа $C_{2}$ зависит только от области.

Ограниченность области $D$ и гладкость ее границ, о которых упоминалось в начале, а также какая-либо регулярность множеств $\Gamma_{\varepsilon} \subset \partial D$ требуются нам лишш в той мере, в какой они необходимы для (2), (3).

Если семейство $\left\{\Gamma_{\varepsilon}\right\}$ самоподобно, то при $\varepsilon \rightarrow 0$ решения $u_{\varepsilon}$ задачи (1) обладают следующими асимптотическими свойствами.

ТеОрема 1. Пусть $g \in L_{s^{\prime}}(\partial D)$, әде $s^{\prime}-$ взаимное число $\kappa$ показателю $s$ из определения $1,1 / s+1 / s^{\prime}=1$. Тогда

1) последовательность $u_{\varepsilon}$ при $\varepsilon \rightarrow 0$ ограничена в пространстве $L_{s}(\partial D)$;

2) существуют измеримая функиия $C: \partial D \rightarrow[0,+\infty)$ и подпоследовательность $\varepsilon_{k} \rightarrow 0$, не зависящие от выбора функиии $g \in L_{s^{\prime}}(\partial D)$, такие, что по этой подпоследовательности функиии $u_{\varepsilon_{k}}$ слабо сходятся $\kappa C(x) g(x)$ в $L_{s}(\partial D)$; 
3) последовательность $u_{\varepsilon}$ компактна в $L_{p}(D)$, а подпоследовательность $u_{\varepsilon_{k}}$ сходится сильно в $L_{p}(D)$ к гармонической функиии и* со значениями $C(x) g(x)$ на граниче $\partial D$ области $D$, где $p<n s /(n-1)$.

ДокАЗАТЕЛЬСтво. Утверждение 3) теоремы вытекает из двух предыдущих в силу следующего свойства гармонических функций.

ПРЕДЛОЖЕНИЕ 1. Пусть D - область с гладкой границей, последовательность гармонических в области $D$ функиий $v_{\varepsilon}$ слабо компактна в $L_{s}(\partial D), s>1$. Тогда она сильно компактна в пространстве $L_{p}(D), p<n s /(n-1)$.

ДоказАтельСтво. Обозначим через $H_{s}^{l}(D), s \neq 1, k<l<k+1(k=0,1, \ldots)$, замькание множества функций из $C^{\infty}(\bar{D})$ по норме

$$
\|v\|_{H_{s}^{l}(D)} \equiv\left(\int_{D \times D} \frac{\left|D^{(k)} v(x+y)-D^{(k)} v(x)\right|^{s}}{|y|^{s+n l}} d x d y\right)^{1 / s} .
$$

Если $l<0$, то по определению $H_{s}^{l} \equiv H_{s^{\prime}}^{-l}$, где $1 / s+1 / s^{\prime}=1$. Теперь воспользуемся неравенством

$$
\|v\|_{L_{p}(D)} \leqslant \widetilde{C}\|v\|_{H_{s}^{l}(D)}, \quad p \leqslant \frac{n s}{n-l s}
$$

из [32] и неравенством

$$
\|v\|_{H_{s}^{l}(D)} \leqslant \widetilde{C}^{1}\|v\|_{H_{s}^{l-1 / s}(\partial D)}, \quad s \neq 1, \quad l>0, \quad l \neq \frac{1}{s},
$$

из [33], которые имеют место для гармонических функций в области $D$.

Из определения соболевских пространств с отрищательньми показателями следует непрерьвность вложения $L_{s}(\partial D) \subset H_{s}^{l-1 / s}(\partial D)$ при $l<1 / s$. Поэтому из неравенства (5) вытекает оценка $\|v\|_{H_{s}^{l}(D)} \leqslant \widetilde{C}^{3}\|v\|_{L_{s}(\partial D)}$. Учитывая неравенство (4), мы получаем оценку $\|v\|_{L_{p}(D)} \leqslant \widetilde{C}^{4}\|v\|_{L_{s}(\partial D)}, p<n s /(n-1)$. Предложение доказано.

Для доказательства утверждения 1$)$ теоремы воспользуемся вариационной формулировкой задачи (1): функции $u_{\varepsilon}$ должны удовлетворять условию Дирихле на $\Gamma_{\varepsilon}$ и интегральному тождеству

$$
\varepsilon \int_{D} \nabla u_{\varepsilon} \nabla v d x=\int_{\partial D} g v d x,
$$

выполненому для любой гладкой функции $v$ с компактным носителем в $\bar{D} \backslash \Gamma_{\varepsilon}$. Через $H_{\varepsilon}$ обозначим замыкание по норме соболевского пространства $W_{2}^{1}(D)$ - пространства гладких функций с компактным носителем в $\bar{D} \backslash \Gamma_{\varepsilon}$. Из (2) и (3) следует, что функционалы в левой и правой частях (6) на $H_{\varepsilon}$ удовлетворяют условиям леммы Лакса-Мильграма (см., например, [34]). Поэтому решение $u_{\varepsilon} \in H_{\varepsilon}$ существует и единственно. Кроме того, по непрерывности соотношения (2), (3), (6) справедливы не только для гладких функций, но и для функций из $H_{\varepsilon}$. При этом из $H_{\varepsilon}$ в $L_{s}(\partial D)$ действует непрерьвный оператор следа, и под значениями функций из $H_{\varepsilon}$ на границе области $D$ подразумеваются значения их следов. 
При $v=u_{\varepsilon}$ из (6) следует неравенство

$$
\varepsilon \int_{D}\left(\nabla u_{\varepsilon}\right)^{2} d x \leqslant\left(\int_{\partial D}|g|^{s^{\prime}} d l\right)^{1 / s^{\prime}}\left(\int_{\partial D}\left|u_{\varepsilon}\right|^{s} d l\right)^{1 / s}
$$

Оценивая правую часть этого неравенства с помощью (2), мы получим оценки

$$
\varepsilon \int_{D}\left(\nabla u_{\varepsilon}\right)^{2} d x \leqslant C_{3}, \quad \int_{\partial D}\left|u_{\varepsilon}\right|^{s} d l \leqslant C_{3}
$$

с константой $C_{3}$, не зависящей от $\varepsilon$. Таким образом, утверждение 1 ) теоремы доказано.

Рассмотрим вспомогательную задачу

$$
\begin{gathered}
\Delta w_{\varepsilon}=0 \text { в } D \\
w_{\varepsilon}=0 \text { на } \Gamma_{\varepsilon}, \\
\varepsilon \frac{\partial w_{\varepsilon}}{\partial \nu}=1 \text { на } \partial D \backslash \Gamma_{\varepsilon},
\end{gathered}
$$

которая совпадает с исходной при $g=1$. Соответствующее этой задаче интегральное тождество в $H_{\varepsilon}$ имеет вид

$$
\varepsilon \int_{D} \nabla w_{\varepsilon} \nabla v d x=\int_{\partial D} v d x
$$

Решение $w_{\varepsilon} \in H_{\varepsilon}$ удовлетворяет оценкам, аналогичным (7):

$$
\varepsilon \int_{D}\left(\nabla w_{\varepsilon}\right)^{2} d x \leqslant C_{4}, \quad \int_{\partial D}\left|w_{\varepsilon}\right|^{s} d x \leqslant C_{4}
$$

с константой $C_{4}$, которая не зависит от $\varepsilon$. Из (10) следует, что можно выбрать подпоследовательность $\varepsilon=\varepsilon_{k}$, по которой следы функций $w_{\varepsilon_{k}}$ слабо сходятся в $L_{s}(\partial D)$. Предельную функцию на $\partial D$ мы обозначим через $C(x)$. Очевидно, что $C \in L_{s}(\partial D)$. Из принципа максимума для гармонических функций следует также, что $C(x) \geqslant 0$.

Выберем произвольную функцию $\theta \in C_{\infty}(\bar{D})$ и в тождества $(6),(9)$ подставим $v=$ $\theta w_{\varepsilon}, v=\theta u_{\varepsilon}$ соответственно. Вычитая одно получившееся равенство из другого, имеем

$$
\varepsilon \int_{D}\left(w_{\varepsilon} \nabla u_{\varepsilon}-u_{\varepsilon} \nabla w_{\varepsilon}\right) \nabla \theta d x=\int_{\partial D}\left(g(x) w_{\varepsilon}-u_{\varepsilon}\right) \theta d x
$$

Покажем, что левая часть (11) стремится к 0 при $\varepsilon \rightarrow 0$. Действительно, из оценок (7), (10) и неравенства Пуанкаре следует, что функции $\sqrt{\varepsilon} u_{\varepsilon}$ и $\sqrt{\varepsilon} w_{\varepsilon}$ ограничены в $W_{2}^{1}(D)$. По теореме Реллиха последовательности этих функций сильно компактны в $L_{2}(D)$, а по норме пространства $L_{p}(D), p<n s /(n-1)$, сходятся, как было доказано вьше, к 0. Поэтому в $L_{2}(D)$ они тоже сходятся к 0 . Таким образом, в произведениях под интегралом в левой части (11) один из сомножителей ограничен в $L_{2}(D)$ при $\varepsilon \rightarrow 0$, а другой сходится к 0 , что и требовалось. 
В правой части (11) перейдем к пределу по подпоследовательности $\varepsilon_{k} \rightarrow 0$. Функции $w_{\varepsilon_{k}}$ слабо сходятся к $C(x)$ в $L_{s}(\partial D)$. Функции $u_{\varepsilon_{k}}$ ограничены в $L_{s}(\partial D)$. Выделим из подпоследовательности $\varepsilon_{k}$ еще одну подпоследовательность, по которой $u_{\varepsilon_{k}}$ слабо сходятся в $L_{s}(\partial D)$ к некоторой предельной функции $u^{*}$ на $\partial D$, и сначала перейдем к пределу по ней. Получим

$$
\int_{\partial D}\left(g(x) C(x)-u^{*}\right) \theta d x=0 .
$$

Ввиду произвольности выбора $\theta \in C_{\infty}(\bar{D})$ на $\partial D$ функция $u^{*}=g C$, т.е. предельная функция $u^{*}$, не зависит от выбора второй подпоследовательности. Поэтому вся последовательность $u_{\varepsilon_{k}}$ имеет единственньй предел. Теорема 1 доказана.

2. Структура случайных множеств $\Gamma_{\varepsilon}$. Для более детального описания семейства $\left\{\Gamma_{\varepsilon}\right\}$ мы используем конструкцию традиционную в теории усреднения (см. [1]). Эта конструкция обладает достаточной степенью общности и годится для описания мелкозернистых множеств с периодической, квазипериодической и случайной структурой.

Пусть $(\Omega, B, \mu)$ - вероятностное пространство, на котором задана полугрупша отображений $T_{x}: \Omega \rightarrow \Omega$, измеримых по $\omega \in \Omega, x \in \mathbb{R}^{n-1}$ и сохраняющих меру $\mu$ на $\Omega$. Предполагается выполненным следующее групповое свойство: для любых $x_{1}, x_{2} \in$ $\mathbb{R}^{n-1}$ и любого $\omega \in \Omega$

$$
T_{x_{1}} \circ T_{x_{2}} \omega=T_{x_{1}+x_{2}} \omega, \quad T_{0} \omega=\omega .
$$

ОПредЕЛЕниЕ 2. Измеримую функцию $\varphi: \Omega \times \mathbb{R}^{n-1} \rightarrow \mathbb{R}$ будем назьвать случайной статистически однородной, если она имеет вид $\varphi=\varphi\left(T_{x} \omega\right)$.

ОПРЕДЕЛЕниЕ 3. Случайное множество назьвается однородным, если однородна его характеристическая функция.

ПРИмЕР. Если $\Omega-(n-1)$-мерный тор с естественной группой сдвигов на нем, то однородность множества и функции означает их периодичность.

Семейство отображений $T$ на $\Omega$ образует динамическую систему с $(n-1)$-мерным “временем" $x$. Всюду далее мы будем считать эту систему әргодической, т.е. любая $\mu$-измеримая функция на $\Omega$, инвариантная относительно этой групшы сдвигов, является почти всюду константой. Это дает нам возможность пользоваться теоремой Биркгофа (см. [1], [35]) в следующей формулировке.

ТЕОРемА БИРКГОФА. Для любой функиии $\varphi \in L_{\alpha}(\Omega), \alpha \geqslant 1$, любой ограниченной области $D \subset \mathbb{R}^{n-1}$ почти наверное выполняется равенство

$$
\lim _{\varepsilon \rightarrow 0} \frac{1}{\operatorname{mes} D} \int_{D} \varphi\left(T_{x / \varepsilon} \omega\right) d x=\int_{\Omega} \varphi(\omega) \mu(d \omega) \equiv\langle\varphi\rangle .
$$

Угловьпи скобками мы обозначаем интеграл по $\Omega$ с мерой $\mu$, т.е. математическое ожидание. Из теоремы Биркгофа следует, что функции $\varphi\left(T_{x / \varepsilon} \omega\right)$ при $\varepsilon \rightarrow 0$ почти наверное слабо сходятся к $\langle\varphi\rangle$ в $L_{\alpha}^{\text {loc }}\left(\mathbb{R}^{n-1}\right)$.

Однородные множества мы будем использовать для построения семейств $\left\{\Gamma_{\varepsilon}\right\}$ мелкомасштабных множеств ячеистой структуры. Если $V(\omega) \in \mathbb{R}^{n-1}$ - однородное множество, то его гомотетические сжатия в $1 / \varepsilon$ раз $\{\varepsilon V(\omega)\}$ образуют такое семейство на 
$(n-1)$-мерном многообразии $\partial D$. В дальнейшем мы будем употреблять одно и то же обозначение $\varepsilon V(\omega)$ для однородного мелкомасштабного множества в $\mathbb{R}^{n-1}$ и для множества в $\mathbb{R}^{n}$, образованного точками $\{(x, z) \in \partial D: x \in \varepsilon V(\omega), z=0\}$, где под $x$ подразумеваются локальные координаты на $\partial D$, а под $z$ - координата вдоль нормали к $\partial D$. Чтобы не усложнять повествование деталями, связанными с криволинейностью локальных координат $(x, z)$ в $\mathbb{R}^{n}$, мы рассмотрим в качестве области $D$ единичный куб $D=\left\{(x, z): 0<x_{i}<1,0<z<1\right\}$ и будем считать, что смешанные граничные условия в (1) заданы лишь на одной грани, а на всех остальных выставлено однородное условие Дирихле. Таким образом, $\Gamma_{\varepsilon}=(\partial D \backslash Q) \cup(Q \cap \varepsilon V(\omega))$, где $Q=\{(x, z): 0<$ $\left.x_{i}<1, z=0\right\}-$ одна из граней куба.

Для того чтобы семейство $\left\{\Gamma_{\varepsilon}\right\}$ было самоподобным в смысле определения 1 , нам потребуются дополнительные свойства однородного множества $V(\omega)$, порождающего это семейство. Потребуем невырожденности $V(\omega)$ (определение 4), что обеспечит самоподобие семейства $\left\{\Gamma_{\varepsilon}\right\}$.

ОПРЕДЕЛЕниЕ 4. Случайное однородное замкнутое множество $V(\omega) \subset \mathbb{R}^{n-1}$ называется невырожденным, если существует положительная статистически однородная функция $h=h(\omega)$ такая, что для почти всех $\omega$ и любой функции $\varphi \in C_{0}^{\infty}\left(\mathbb{R}^{n} \backslash V(\omega)\right)$ с компактным носителем, не содержащим $V(\omega)$, вьполнено неравенство

$$
\int_{\mathbb{R}^{n-1}} h\left(T_{x} \omega\right) \varphi^{2}(x, 0) d x \leqslant \int_{0}^{\infty} d z \int_{\mathbb{R}^{n-1}}|\nabla \varphi(x, z)|^{2} d x
$$

при этом

$$
\left\langle h^{-(1+\delta)}\right\rangle<+\infty
$$

с некоторым $\delta, 0<\delta \leqslant+\infty$.

Условие невырожденности $V(\omega)$ будет использовано ниже для исследования вспомогательной задачи (16). Эта задача сводится к некоторому псевдодифференциальному уравнению в области $\mathbb{R}^{n-1} \backslash V$. Оценки $(12),(13)$ гарантируют ее разрешимость. В [29], [30] похожие условия использовались для доказательства разрешимости некоторых уравнений в случайных неограниченных областях в связи с проблемой усреднения течений вязкой жидкости в пористой среде.

При $h=$ const $>0$ неравенство (12) фактически означает непрерывность вложения $H^{1 / 2}\left(\mathbb{R}^{n-1} \backslash V(\omega)\right)$ в $L_{2}\left(\mathbb{R}^{n-1} \backslash V(\omega)\right)$. Для ограниченных областей непрерывность этого вложения имеет место (см. [3], [36]), а для неограниченных ее может и не быть. Если, например, область $\mathbb{R}^{n-1} \backslash V(\omega)$ содержит шар большого радиуса $R$, то из соображений подобия нетрудно установить, что константа $h$ в неравенстве (12) по порядку величины не должна превьшать $1 / R$. Конкретные модели случайных множеств конструируются, как правило, так, что для любого $R>0$ найдется шар радиуса $R$ в $\mathbb{R}^{n-1}$, не пересекающийся с $V(\omega) \subset \mathbb{R}^{n-1}$. Очевидно, что в этом случае неравенство (12) с $h=$ const не вьполняется. Однако оно может иметь место, если весовая функция $h\left(T_{x} \omega\right)$ будет принимать значения тем меньшие, чем более точка $x \in \mathbb{R}^{n-1}$ удалена от $V(\omega)$. При этом, вообше говоря, может нарушаться неравенство (13), так как функция $h^{-1}$ оказьвается неограниченной. 
Ниже мы приведем способ построения весовой функции $h$, пригодный для весьма широкого класса моделей случайных множеств.

Пусть область $V(\omega)$ получается объединением в $\mathbb{R}^{n-1}$ шаров радиусов $\rho_{i}>0$ с центрами в изолированных точках $y_{i}$. Шарам разрешается перекрьваться друг с другом. Введем следующие обозначения. Пусть $\widetilde{r}=r_{\omega}(y)-$ расстояние от точки $y \in \mathbb{R}^{n-1}$ до ближайшего центра $y_{i}, \widetilde{\rho}=\rho_{\omega}(y)$ - радиус шара с центром $y_{i}$, ближайшим к $y$. Если $V(\omega)$ - статистически однородная область, то функции $\widetilde{r}$ и $\widetilde{\rho}$ тоже статистически однородны.

Они являются локальными характеристиками случайного множества $V(\omega)$, статистические свойства которых для большинства конкретных моделей случайных структур легко контролируются. Мы выразим $h$ из (12) через функции $\widetilde{r}$ и $\widetilde{\rho}$. Таким образом, неравенство (13) в определении 4 станет эффективно проверяемым.

ПРЕДЛОЖЕНИЕ 2. Неравенство (12) выполняется, если в качестве $h$ взять функцию

$$
h=\frac{1}{\widetilde{\rho}} H\left(\frac{\widetilde{r}}{\widetilde{\rho}}\right), \quad H(t)= \begin{cases}\frac{1}{2 t^{3}} & \text { npu } n=2, \\ \frac{1}{8 t^{3} \ln (t+1)} & \text { npu } n=3, \\ \frac{n-3}{4 t^{2 n-3}} & \text { npu } n>3 .\end{cases}
$$

ДокАЗАтельство. Разобьем $\mathbb{R}^{n-1}$ на измеримые подмножества $V_{i}$, состоящие из точек, для которых $y_{i}-$ ближайший центр. Если множество центров $\left\{y_{i}\right\}$ не имеет точек накопления, то $V_{i}$ - это некоторые полиэдры. В каждом из них введем полярные координаты $(r, \theta)$, где $r=\left|y-y_{i}\right|, \theta$ - полярные углы. Полиэдры звездны относительно центра, поэтому их границы задаются в полярных координатах однозначными функциями $r=R(\theta)$. Внутри каждого полиэдра функция $\rho_{\omega}(y)$ принимает постоянное значение $\widetilde{\rho}>0$.

Для каждой точки $M \in V_{i}$ с координатами $(\widetilde{r}, \theta), \widetilde{r}>\widetilde{\rho}$, положим $\widetilde{a}=\widetilde{\rho}^{2} / \widetilde{r}$ и построим точку $\widetilde{M} \in V_{i}$ с координатами $(\widetilde{a}, \theta), 0<\widetilde{a}<\widetilde{\rho}$. Соединим точки $M$ и $\widetilde{M}$ кривой $l$ в цилиндре $V_{i} \times[0, \infty)$, которая задается в цилиндрических координатах $(r, \theta, z)$ уравнением

$$
z=\frac{(\widetilde{r}-r)(r-\widetilde{a})}{\widetilde{r}-\widetilde{a}}, \quad \theta=\mathrm{const}, \quad \widetilde{a} \leqslant r \leqslant \widetilde{r}
$$

Рассмотрим произвольную функцию $\varphi \in C_{0}^{\infty}\left(\mathbb{R}^{n} \backslash V(\omega)\right)$ с компактным носителем, для которой необходимо проверить неравенство (12). В каждом цилиндре $V_{i} \times[0, \infty)$ $\varphi(r, \theta, 0) \equiv 0$ при $r<\widetilde{\rho}$. Представим значение $\varphi$ в точке $(\widetilde{r}, \theta, 0), \widetilde{r}>\widetilde{\rho}$, в виде интеграла вдоль кривой $l$ :

$$
\varphi(\widetilde{r}, \theta, 0)=\int_{\widetilde{a}}^{\widetilde{r}} \frac{d \varphi}{d r} d r
$$

где

$$
\frac{d \varphi}{d r}=\frac{\partial \varphi}{\partial r}+\frac{\partial \varphi}{\partial z} \frac{d z}{d r}
$$


- производная вдоль кривой $l$. Очевидно,

$$
\left|\frac{d \varphi}{d r}\right| \leqslant|\nabla \varphi| \sqrt{1+\left(\frac{d z}{d r}\right)^{2}}
$$

Используя неравенство Шварца-Коши-Буняковского, получим

$$
\varphi^{2}(\widetilde{r}, \theta, 0) \leqslant \int_{\widetilde{a}}^{\widetilde{r}}|\nabla \varphi|^{2}\left(1+\left(\frac{d z}{d r}\right)^{2}\right) r^{n-2} d r \int_{\widetilde{a}}^{\widetilde{r}} \frac{d t}{t^{n-2}} .
$$

Введем обозначение

$$
I=I(\theta)=\int_{\widetilde{\rho}}^{R(\theta)} \varphi^{2}(\widetilde{r}, \theta, 0) \frac{1}{\widetilde{\rho}} H\left(\frac{\widetilde{r}}{\widetilde{\rho}}\right) \widetilde{r}^{n-2} d \widetilde{r} .
$$

После интегрирования $I(\theta)$ по полярным углам и суммирования по всем полиэдрам $V_{i}$ получится левая часть доказываемого неравенства (12). Из (14) получим оценку

$$
I \leqslant \int_{\widetilde{\rho}}^{R(\theta)}\left(\int_{\widetilde{a}}^{\widetilde{r}}\left(|\nabla \varphi|^{2}\left(1+\left(\frac{d z}{d r}\right)^{2}\right) r^{n-2} \frac{1}{\widetilde{\rho}} H\left(\frac{\widetilde{r}}{\widetilde{\rho}}\right) \widetilde{r}^{n-2}\left(\int_{\widetilde{a}}^{\widetilde{r}} \frac{d t}{t^{n-2}}\right)\right) d r\right) d \widetilde{r} .
$$

В этой оценке мы перейдем от переменных интегрирования $(\widetilde{r}, r)$ к переменным $(r, z)$. Якобиан перехода имеет вид

$$
\frac{d z}{d \widetilde{r}}=\frac{\widetilde{r}(r-\widetilde{a})^{2}+\widetilde{a}(\widetilde{r}-r)^{2}}{\widetilde{r}(\widetilde{r}-\widetilde{a})^{2}}>0
$$

Прямыми вычислениями устанавливаются неравенства

$$
1+\left.\left(\frac{d z}{d r}\right)^{2}\right|_{\tilde{a} \leqslant r \leqslant \widetilde{r}} \leqslant 2,\left.\quad \frac{d z}{d \widetilde{r}}\right|_{\tilde{a} \leqslant r \leqslant \widetilde{r}} \geqslant \frac{\widetilde{a}}{\widetilde{r}+\widetilde{a}}
$$

Таким образом,

$$
I \leqslant 2 \int_{\widetilde{\rho}}^{R}\left(\int_{\widetilde{a}}^{\widetilde{r}}\left(|\nabla \varphi|^{2} r^{n-2} \frac{d z}{d \widetilde{r}} \max _{\tilde{r}, \tilde{\rho} \leqslant \widetilde{r} \leqslant R}\left\{\frac{1}{\widetilde{\rho}} H\left(\frac{\widetilde{r}}{\widetilde{\rho}}\right) \widetilde{r}^{n-2}\left(\frac{\widetilde{a}}{\widetilde{r}+\widetilde{a}}\right)^{-1} \int_{\widetilde{a}}^{\widetilde{r}} \frac{d t}{t^{n-2}}\right\}\right) d r\right) d \widetilde{r} .
$$

$\Phi$ ункция $H(t)$ выбрана так, что

$$
2 \max _{\tilde{r}, \tilde{\rho} \leqslant \widetilde{r} \leqslant R}\left\{\frac{1}{\widetilde{\rho}} H\left(\frac{\widetilde{r}}{\widetilde{\rho}}\right) \widetilde{r}^{n-2}\left(\frac{\widetilde{a}}{\widetilde{r}+\widetilde{a}}\right)^{-1} \int_{\widetilde{a}}^{\widetilde{r}} \frac{d t}{t^{n-2}}\right\} \leqslant 1
$$

для всех $\widetilde{\rho}$ и $R$. Учитывая это при переходе от $(\widetilde{r}, r)$ к $(z, r)$ и увеличивая область интегрирования, получим

$$
I \leqslant \int_{\widetilde{\rho}}^{R(\theta)}\left(\int_{\widetilde{a}}^{\widetilde{r}}\left(|\nabla \varphi|^{2} r^{n-2} \frac{d z}{d \widetilde{r}}\right) d r\right) d \widetilde{r} \leqslant \int_{0}^{\infty}\left(\int_{0}^{R(\theta)}|\nabla \varphi|^{2} r^{n-2} d r\right) d z .
$$

После интегрирования по полярньп углам и суммирования по $i$ получаем (12), что и требовалось. 
3. Теорема усреднения. Если семейство мелкомасштабных множеств $\Gamma_{\varepsilon} \subset \partial D$ порождено невырожденным однородным множеством $V(\omega)$, то асимптотика решений $u_{\varepsilon}$ задачи (1) может быть описана более подробно, чем в теореме 1. Напомним, что мы рассматриваем в качестве области $D$ единичный куб, $\Gamma_{\varepsilon}=(\partial D \backslash Q) \cup(Q \cap \varepsilon V(\omega))$ и $Q-$ одна из граней куба.

Теорема 2. Если $V(\omega)$ - невырожденное замкнутое множество с показателем $\delta>0$ в определении 4 , то почти наверное $\left\{\Gamma_{\varepsilon}\right\}$ - самоподобное семейство $c$ показателем $s=1+\delta /(2+\delta)$ и для последовательности решений $u_{\varepsilon}$ задачи (1) справедливы утверждения теоремы 1. При этом предельная функиия $u^{*}$ единственна и неслучайна. Граничная функиия $C(x)$ не зависит от выбора подпоследовательности, обращается в 0 на $\partial D \backslash Q$, а на $Q$ равна положительной константе.

ДокАЗАтЕльство. Сначала докажем самоподобие семейства $\left\{\Gamma_{\varepsilon}\right\}$. Пусть $\varphi-$ гладкая функция в $\bar{D}$ с носителем в $\bar{D} \backslash \Gamma_{\varepsilon}$. Положим $s=1+\delta /(2+\delta), \alpha=2 / s, \beta=2 /(2-s)$, так что $1 / \alpha+1 / \beta=1$. В силу неравенства Гёльдера имеем

$$
\int_{Q}|\varphi|^{s} d x \leqslant\left(\int_{Q} h\left(T_{x / \varepsilon} \omega\right)|\varphi|^{2} d x\right)^{1 / \alpha}\left(\int_{Q} h^{-\beta / \alpha}\left(T_{x / \varepsilon} \omega\right) d x\right)^{1 / \beta}
$$

где $Q$ - та грань куба $D$, в которой содержится множество $\partial D \backslash \Gamma_{\varepsilon}$, а $h(\omega)-$ весовая функция из определения невырожденности 4.

В неравенстве (12) произведем масштабноепреобразованиепеременных $(x, z) \mapsto(x / \varepsilon$, $z / \varepsilon)$. Тогда первьй сомножитель в правой части (15) оценивается интегралом по кубу $D$ :

$$
\int_{Q} h\left(T_{x / \varepsilon} \omega\right) \varphi^{2}(x, 0) d x \leqslant \varepsilon \int_{D}|\nabla \varphi|^{2} d x d z
$$

Второй же сомножитель вследствие теоремы Биркгофа почти наверное имеет конечный предел равньй $\left\langle h^{-(1+\delta)}\right\rangle^{1 / \beta}$, так как $\beta / \alpha=1+\delta$. Таким образом, из (15) вытекает неравенство (2). Это означает, что с вероятностью 1 семейство $\left\{\Gamma_{\varepsilon}\right\}$ самоподобно и для решений $u_{\varepsilon}$ справедливы утверждения теоремы 1 .

Граничная функция $C(x)$ является предельной для последовательности решений $w_{\varepsilon}$ вспомогательной задачи (8). В условиях теоремы 2 на $\partial D \backslash Q w_{\varepsilon}=0$, поэтому на всех гранях куба $D$, кроме $Q, C(x) \equiv 0$ независимо от выбора подпоследовательности $\varepsilon_{k} \rightarrow$ 0 . Осталось показать, что на $Q$ граничная функция $C(x)$ определяется единственным образом и равна положительной неслучайной константе.

Наряду с (8) рассмотрим следующую вспомогательную задачу в $\mathbb{R}_{+}^{n}=\mathbb{R}^{n-1} \times[0,+\infty)$ :

$$
\begin{gathered}
\sum_{i=1}^{n-1} \frac{\partial^{2} W}{\partial x_{i}^{2}}+\frac{\partial^{2} W}{\partial z^{2}}=0 \text { в } \mathbb{R}_{+}^{n}, \\
W=0 \text { на } V(\omega), \\
\frac{\partial W}{\partial z}=-1 \text { на } \mathbb{R}^{n-1} \backslash V(\omega) .
\end{gathered}
$$


Уравнения (16) соответствуют вспомогательной задаче (8) и формально получены из нее заменой $(x, z) \mapsto(x / \varepsilon, z / \varepsilon)$. Более существенньм отличием является неограниченность области $\mathbb{R}_{+}^{n}$. Опишем функциональное пространство, в котором мы будем искать решения (16).

Пусть $\boldsymbol{S}$ - линейное пространство функций $W: \Omega \times[0,+\infty) \rightarrow \mathbb{R}$, реализации $W\left(T_{x} \omega, z\right)$ которых суть гладкие функции в $\mathbb{R}_{+}^{n}$, равномерно по $\omega \in \Omega$ ограниченные вместе с производньми и имеющие носитель в $\mathbb{R}_{+}^{n} \backslash V(\omega)$, ограниченньй в направлении $z$. На $\boldsymbol{S}$ определен оператор, соответствующий задаче (16). Решение этой задачи будем искать на замыкании $\boldsymbol{S}$ в подходящей норме.

Для невырожденных областей $V(\omega)$ функции из $\boldsymbol{S}$ обладают следующими свойствами.

ЛЕмма 1. Для любой функции $W \in \boldsymbol{S}$ выполнены неравенства

$$
\begin{aligned}
\left\langle W^{2}(\omega, 0) h(\omega)\right\rangle & \leqslant \int_{0}^{\infty}\left\langle\left|\nabla W\left(T_{x} \omega, z\right)\right|^{2}\right\rangle d z \\
\left\langle|W(\omega, 0)|^{t}\right\rangle^{1 / t} & \leqslant C(t)\left\langle W^{2}(\omega, 0) h(\omega)\right\rangle^{1 / 2}
\end{aligned}
$$

где $h(\omega)$ - весовая функиия из определения невырохденности $4, t$ - постоянное число, $1 \leqslant t \leqslant s=1+\delta /(2+\delta)$, , $C(t)-$ полохительная константа, $C(1)=$ $\left\langle h^{-1}\right\rangle^{1 / 2}$.

Доказательство леммы 1 будет дано ниже. Отметим, что вследствие инвариантности вероятностной меры $\mu$ относительно сдвигов $T_{x}$ выражение в правой части (17) не зависит от $x$. Это выражение обладает свойствами квадрата нормы, и замькание пространства $\boldsymbol{S}$ по этой норме мы обозначим через $\overline{\boldsymbol{S}}$. Неравенство (18) показьвает, что для функций $W \in \overline{\boldsymbol{S}}$ корректно определен след $W(\omega, 0)$ и оператор следа непрерьвно действует из $\overline{\boldsymbol{S}}$ в $L_{s}(\partial \Omega)$.

Решением вспомогательной задачи (16) мы назовем реализацию $W\left(T_{x} \omega, z\right)$ функции $W \in \overline{\boldsymbol{S}}$, удовлетворяющую интегральному тождеству

$$
\int_{0}^{\infty}\left\langle\nabla W\left(T_{x} \omega, z\right) \nabla W^{\prime}\left(T_{x} \omega, z\right)\right\rangle d z=\left\langle W^{\prime}\left(T_{x} \omega, 0\right)\right\rangle
$$

для любой функции $W^{\prime} \in \overline{\boldsymbol{S}}$. Это тождесво получается, если формально умножить уравнение Лапласа в $(16)$ на $W^{\prime}\left(T_{x} \omega, z\right)$, проинтегрировать полученное равенство по $\Omega \times[0, \infty)$ и перебросить производные с $W$ на $W^{\prime}$ в соответствии с правилами интегрирования по частям.

Из леммы 1 следует, что билинейная форма и линейньй функционал в (19) удовлетворяют условиям леммы Лакса-Мильграма. Таким образом, обобщенное решение $W \in \overline{\boldsymbol{S}}$ задачи (16) существует и единственно. Кроме того, при $W=W^{\prime}$ из (19) получается оценка

$$
\langle W(\omega, 0)\rangle \leqslant\left\langle h^{-1}\right\rangle .
$$

Реализации $W\left(T_{x} \omega, z\right)$ обобщенного решения удовлетворяют уравнениям (16) не только в смысле $(19)$, но и локально как функции из $W_{\mathrm{loc}}^{1,2}\left(\mathbb{R}_{+}^{n}\right)$. 
ПРЕДЛОЖЕНИЕ 3. Для почти всех $\omega \in \Omega$ реализачии $W\left(T_{x} \omega, z\right)$ решения задачи (16) принадлежсат $W_{\mathrm{loc}}^{1,2}\left(\mathbb{R}_{+}^{n}\right)$ и удовлетворяют интегральному тожсдеству

$$
\int_{\mathbb{R}_{+}^{n}} \nabla W\left(T_{x} \omega, z\right) \nabla \psi(x, z) d x d z=\int_{\mathbb{R}^{n-1}} \psi(x, 0) d x
$$

для любой гладкой функиии $\psi(x, z)$ с компактным носителем в $\mathbb{R}_{+}^{n} \backslash V(\omega)$.

Доказательство похожего утверждения, применимого в более общих ситуациях, можно найти в [1], и мы его не приводим. В рассматриваемом случае можно показать, что с вероятностью 1 (почти наверное) функции $W\left(T_{x} \omega, z\right)$ бесконечно дифференцируемы в $\mathbb{R}_{+}^{n} \backslash V(\omega)$ и удовлетворяют в обычном смысле как уравнению Лапласа, так и условию Неймана в (16).

Нам потребуется тождество (21) в несколько измененном виде:

$$
\varepsilon \int_{\mathbb{R}_{+}^{n}} \nabla W\left(T_{x / \varepsilon} \omega, \frac{z}{\varepsilon}\right) \nabla v(x, z) d x d z=\int_{\mathbb{R}^{n-1}} v(x, 0) d x
$$

Здесь $v(x, z)$ - гладкая функция с носителем в $\mathbb{R}_{+}^{n} \backslash \varepsilon V(\omega)$. Тождество (22) получено из (21) масштабным преобразованием координат.

Пусть $\theta \in C^{\infty}(\bar{D})$ - гладкая функция в кубе $D$ с носителем, содержашимся в $D \cup Q$ (т.е. $\theta \equiv 0$ на всех гранях куба, кроме $Q$ ). В тождество $(22)$ подставим $v=\theta(x, z) w_{\varepsilon}(x, z)$, где $w_{\varepsilon}$ - решение задачи (8), а в тождестве (9) положим $v=\theta(x, z) W\left(T_{x / \varepsilon} \omega, z / \varepsilon\right)$. Вычитая одно полученное равенство из другого, имеем

$$
\begin{gathered}
\varepsilon \int_{D}\left(w_{\varepsilon}(x, z) \nabla W\left(T_{x / \varepsilon} \omega, \frac{z}{\varepsilon}\right)-W\left(T_{x / \varepsilon} \omega, \frac{z}{\varepsilon}\right) \nabla w_{\varepsilon}(x, z)\right) \theta(x, z) d x d z \\
=\int_{Q}\left(w_{\varepsilon}(x, 0)-W\left(T_{x / \varepsilon} \omega, \frac{z}{\varepsilon}\right)\right) \theta(x, 0) d x
\end{gathered}
$$

Перейдем к пределу при $\varepsilon \rightarrow 0$. Левая часть стремится к 0 аналогично левой части равенства (11) в доказательстве теоремы 1 . В правой части функции $W\left(T_{x / \varepsilon} \omega, 0\right)$ слабо сходятся к $\langle W(\omega, 0)\rangle$ в $L_{s}(Q)$ вследствие эргодичности, а функции $w_{\varepsilon}(x, 0)$ сходятся к $C(x)$ по некоторой подпоследовательности $\varepsilon_{k} \rightarrow 0$ по теореме 1 . Так как $\theta(x, z)-$ произвольная функция, на $Q$ вьполнено $C(x) \equiv\langle W(\cdot, 0)\rangle$ независимо от выбора подпоследовательности. Следовательно, вся последовательность $w_{\varepsilon}(x, 0)$ на $Q$ сходится к этому неслучайному пределу. Полагая в тождестве $(19) W^{\prime}=W$, получим, что $\langle W(\cdot, 0)\rangle \geqslant 0$. Равенство имеет место в вырожденном случае, когда линейный функционал в правой части (19) равен 0 для всех $W^{\prime} \in \overline{\boldsymbol{S}}$, т.е. когда множество $V(\omega)$ для почти всех $\omega$ совпадает с $\mathbb{R}^{n-1}$. Для $C(x)$ справедлива также оценка сверху (20).

Таким образом, для завершения доказательства теоремы 2 осталось обосновать лемmy 1. 
ДокАЗАТЕЛЬСТво ЛЕммы 1 . Пусть $B$ и $B_{1}-$ шары в $\mathbb{R}^{n-1}$ с общим центром и радиусами $R$ и $R(1+r), r>0$, соответственно. Построим гладкую срезающую функцию $\psi \in C_{0}^{\infty}\left(\mathbb{R}^{n-1}\right)$ с носителем в $B_{1}$ такую, что $\psi \equiv 1$ на $B,|\psi| \leqslant 1,|\nabla \psi| \leqslant 2 /(r R)$. В неравенстве $(12)$ положим $\varphi(x, z)=\psi(x) W\left(T_{x} \omega, z\right), W \in \boldsymbol{S}$. Получим

$$
\int_{B_{1}} h\left(T_{x} \omega\right) \psi^{2}(x) W^{2}\left(T_{x} \omega, 0\right) d x \leqslant \int_{0}^{\infty} \int_{B_{1}}\left|\nabla\left(\psi(x) W\left(T_{x} \omega, z\right)\right)\right|^{2} d x d z
$$

откуда следует оценка

$$
\begin{aligned}
& \int_{B} h\left(T_{x} \omega\right) W^{2}\left(T_{x} \omega, 0\right) d x \\
& \quad \leqslant \int_{0}^{\infty} \int_{B_{1}}\left((1+m)\left|\nabla W\left(T_{x} \omega, z\right)\right|^{2}+\left(\frac{2}{r R}\right)^{2}\left(1+\frac{1}{m}\right) W^{2}\left(T_{x} \omega, z\right)\right) d x d z .
\end{aligned}
$$

Мы воспользовались очевидным неравенством $(a+b)^{2} \leqslant(1+m) a^{2}+(1+1 / m) b^{2}$ для произвольного $m>0$ и свойствами срезающей функции $\psi$. Поделим обе части неравенства на объем шара $B$ и перейдем к пределу при $R \rightarrow \infty$. По эргодической теореме эти пределы существуют почти наверное. Получим

$$
\left\langle h(\cdot) W^{2}(\cdot, 0)\right\rangle \leqslant(1+m)(1+r)^{n-1} \int_{0}^{\infty}\left\langle\mid \nabla W\left(T_{x} \cdot, z\right)\right\rangle d z .
$$

Отсюда, устремляя параметры $m$ и $r$ к 0, получим (17). Оценка (18) получается в результате применения неравенства Гёльдера:

$$
\left\langle|W(\cdot, 0)|^{t}\right\rangle=\left\langle|W(\cdot, 0)|^{t} h^{t / 2}(\cdot) h^{-t / 2}(\cdot)\right\rangle \leqslant\left\langle W^{2}(\cdot, 0) h(\cdot)\right\rangle^{t / 2}\left\langle h^{-t /(2-t)}(\cdot)\right\rangle^{(2-t) / 2} .
$$

Поскольку $t /(2-t) \leqslant 1+\delta$, второй сомножитель правой части неравенства конечен согласно (13). Лемма 1 доказана.

Теперь доказательство теоремы 2 завершено полностью.

Авторы очень признательны профессору В. А. Кондратьеву за предоставленное доказательство предложения 1 о сильной компактности последовательности гармонических функций. 


\section{СПИСОК ЦИТИРОВАННОЙ ЛИТЕРАТУРЫ}

[1] Жииков В. В., Козлов С. М., Олейник О. А. Усреднение дифференциальных операторов. М.: Физматлит, 1993.

[2] Марченко В.А., Хруслов Е. Я. Краевые задачи в областях с мелкозернистой границей. Киев: Наукова думка, 1974.

[3] Санчес-Паленсия Э. Неоднородные среды и теория колебаний. М.: Мир, 1984.

[4] Беляев А.Г. Усреднение третьей краевой задачи для уравнения Пуассона в области с быстро осциллирующей границей // Вестн. МГУ. Сер. 1. Матем., мех. 1988. №6. С. 63-66.

[5] Беляев А. Г. О сингулярных возмущениях краевых задач. Дисс. ... к. ф.-м.н. М.: МГУ, 1990.

[6] Bouchitte G., Lidouh A., Michel J. C., Suquet P. Might Boundary Homogenization Help to Understand Friction? SMR 719/9. Trieste: Internat. Centre for Theoretical Physics, 1993.

[7] Bouchitte G., Lidouh A., Suquet P. Homogénéisation de frontière pour la modélisation du contact entre un corps déformable non-linéaire et un corps rigide // C. R. Acad. Sci. Paris. Sér. I. Math. 1991. V. 313. P. 967-972.

[8] Беляев А. Г., Михеев А. Г., Шамаев А.С. Дифракция плоской волны на быстро осциллирующей поверхности // ЖВМиМФ. 1992. Т. 32. № 8. С. 1258-1272.

[9] Мовчан А. Б., Назаров С. А. Влияние малых неровностей поверхности на напряженное состояние тела и энергетический баланс при росте трешины // ПММ. 1991. Т. 55. № 5 . C. 819-828.

[10] Oleinik O. A., Shamaev A. S., Yosifian G. A. Mathematical Problems in Elasticity and Homogenization. Amsterdam: North-Holland, 1992.

[11] Пятницкий А. Л., Чечкин Г.А. О задаче Неймана в областях с быстрым нарастанием осцилляции границы // УМН. 1996. Т. 51. № 5. С. 166-167.

[12] Sanchez-Palencia E. Homogenization Techniques for Composite Media. Berlin-New York: Springer, 1987.

[13] Sanchez-Palencia E., Suquet P. Friction and homogenization of a boundary // Free Boundary Problems: Theory and Applications / ed. A. Fasano, M. Primicerio. London: Pitman, 1983. P. 561-571.

[14] Chechkin G. A., Friedman A., Piatnitski A. L. The Boundary Value Problem in Domains with Very Rapidly Oscillating Boundary. INRIA Rapport de Recherche № 3062 (Décembre 1996). Sophia Antipolis: Unité de Recherche, Institut National de Recherche en Informatique et en Automatique, 1996.

[15] Belyaev A. G., Chechkin G.A., Piatnitski A. L. Asymptotic Behavior of Solutions for Boundary Value Problems in a Perforated Domain with Oscillating Boundary. Publications du Laboratoire d'Analyse. № R98000 (July 1998). Paris: Université P. et M. Curie, CNRS, 1998.

[16] Беляев А. Г., Плтницкий А. Л., Чечкин Г.А. Асимптотическое поведение решения краевой задачи в перфорированной области с осциллирующей границей // Сиб. матем. ж. 1998. T. 39. № 4. C. $730-754$.

[17] Butazzo G., Dal Maso G., Mosco U. Asymptotic Behavior for Dirichlet Problems in Domains Bounded by Thin Layers. Preprint di Matematica № 2. Pisa: Scuola Norm. Sup., 1987.

[18] Чечкин Г.А. Усреднение краевых задач с сингулярным возмущением граничных условий // Матем. сб. 1993. Т. 184. №6. С. 99-150.

[19] Чечкин Г. А. О краевых задачах для эллиптического уравнения второго порядка с осциллирующими граничными условиями // Неклассические дифференциальные уравнения в частных производных. Новосибирск: ИМ СО АН СССР, 1988. С. 95-104.

[20] Чечкин Г. А. Об асимптотических свойствах частично закрепленной мембраны // УМН. 1989. T. 44. № 4. C. 227.

[21] Олейник О. А., Чечкин Г. А. О краевых задачах для эллиптических уравнений с быстро меняющимся типом граничных условий // УМН. 1993. Т. 48. №6. С. 163-164. 
[22] Олейник О. А., Чечкин Г. А. О краевых задачах для системы теории упругости с быстро меняющимся типом граничных условий // УМН. 1994. Т. 49. № 4. С. 114.

[23] Чечкин Г. А. Полное асимптотическое разложение решения краевой задачи с быстро меняющимися граничными условиями в слое // УМН. 1993. Т. 48. № 4. С. 218-219.

[24] Чечкин Г. А. О колебаниях тел с концентрированными массами, расположенными на границе // УМН. 1995. Т. 50. № 4. С. 105-106.

[25] Чечкин Г. А. Асимптотическое разложение решения краевой задачи с быстро меняющимся типом граничных условий // Тр. семинара им. И. Г. Петровского. 1996. Т. 19. С. 323-337.

[26] Беляев А. Г., Чечкин Г. А. Усреднение смешанной краевой задачи для оператора Лапласа в случае, когда предельная задача неразрешима // Матем. сб. 1995. Т. 186. № 4. С. 47-60.

[27] Олейник О. А., Чечкин Г. А. О поведении решений и собственных значений краевых задач с быстро меняющимися граничными условиями для системы теории упругости // Современные проблемы механики сплошной среды. Ростов-на-Дону: МП "Книга", 1995. С. 167-172.

[28] Chechkin G.A., Oleinik O.A. On the asymptotics of solutions and eigenvalues of the boundary value problems with rapidly alternating boundary conditions for the system of elasticity // Atti Accad. Naz. Lincei. Cl. Sci. Fis. Mat. Natur. Rend. Lincei (9). Mat. Appl. 1996. V. 7. № 1. P. 5-15.

[29] Беляев А. Ю., Козлов С. М. Усреднение уравнений Стокса в случайной перфорированной области // УМН. 1993. Т. 48. № 4. С. 211-212.

[30] Beliaev A. Yu., Kozlov S. M. Darcy equation for random porous media // Comm. Pure Appl. Math. 1996. V. 49. № 1. P. 1-34.

[31] Чечкин Г. А., Беляев А. Ю. Случайное граничное усреднение // Международная конференция "Функциональные пространства, теория приближений, нелинейный анализ" (г. Москва, 27 апреля - 3 мая 1995 г.). Тезисы докл. М., 1995. С. 296-298.

[32] Бесов О.В., Ильин В. П., Никольский С. М. Интегральные представления функций и теоремы вложения. М.: Наука, 1975.

[33] Ройтберг Я.А., Шефтель З. Г. Теорема о гомеоморфизмах для эллиптических систем и ее приложения // Матем. сб. 1969. Т. 78(120). №3. С. 446-472.

[34] Iosida K. Functional Analysis. New York: Springer, 1965.

[35] Данфорд Н., Шварц Дж. Т. Линейные операторы. Общая теория. М.: ИЛ, 1962.

[36] Темам Р. Уравнение Навье-Стокса. Теория и численный анализ. М.: Мир, 1981.

Институт водных проблем РАН

Поступило

Московский государственный университет им. М. В. Ломоносова 08.05 .96

E-mail: chechkin@mech.math.msu.su

Исправленный вариант 30.09 .98 